\title{
Evaluation of Bone Mineral Density by Dual Energy X-ray Absorptiometry in Patients with Chronic Obstructive Pulmonary Disease
}

\author{
Nafisa Jahan ${ }^{1}$, K. B. M. Abdur Rahman ${ }^{2}$, Nadiruzzaman ${ }^{1}$ \\ ${ }^{1}$ Institute of Nuclear Medicine and Allied Sciences, Barisal, ${ }^{2}$ Director Bioscience, Dhaka \\ Address of Correspondence: Nafisa Jahan, MBBS, M Phil (Nuclear Medicine), Director, \\ Institute of Nuclear Medicine and Allied Sciences, Barisal- 8200. Bangladesh, E-mail: nafisajahan@gmail.com
}

\begin{abstract}
Introduction: Osteoporosis or low bone mass is one of the systemic effects of the chronic obstructive pulmonary disease (COPD). Fracture may occur as a complication of low bone mass in these patients that increases the morbidity. There is lack of awareness about the decreased bone mass in these patients. There is significant need to screen those patients who are at risk, so that early diagnosis can be made and preventive and or therapeutic measures can be taken to avoid the consequences of osteoporosis. Dual energy X-ray absorptiometry (DEXA) is the current gold standard for the diagnosis of osteoporosis or low bone mass.
\end{abstract}

Objective: To evaluate the bone mineral density of the chronic obstructive pulmonary disease patients with dual energy X-ray absorptiometry and compare with the controls (non-COPD patients).

Method: In the period of January 2010 to December 2010 a total number of 100 subjects were included in the study. Among them fifty patients were with chronic obstructive pulmonary disease and fifty apparently healthy persons without chronic lung diseases taken as comparison group controls. Bone mineral density (BMD) of lower lumber spine (L1-L4) and left femoral neck were measured by Lunar DPX pro (GE) scanner. Low BMD was defined according to World Health Organization (WHO) criteria based on $T$ score of patients and were compared with the controls.

Results: BMD was significantly low in COPD patients than in control group. At femoral neck, low BMD was found among $84 \%$ of patients with COPD and $36 \%$ of controls without COPD. At lumber spine low BMD was found among $86 \%$ of patients with COPD and $60 \%$ of patients without COPD.

Conclusion: This study suggests that bone mineral density is low in chronic obstructive pulmonary disease.

Key words: Chronic obstructive pulmonary disease (COPD); Bone mineral density (BMD), Dual energy $X$-ray absorptiometry (DEXA), Osteoporosis.

\section{INTRODUCTION}

Chronic obstructive pulmonary disease (COPD) is a heterogeneous condition embracing several overlapping pathological processes including chronic bronchitis, chronic bronchiolitis and emphysema. COPD is an increasing problem in both the Western and developing world \& causes significant morbidity and disability. COPD is gaining global importance due to the marked increase in tobacco consumption in the developing countries. If current trend continue, it will become the third most common cause of death worldwide by 2020 (1). COPD is a common disease in Bangladesh. In the general population of our country, prevalence of COPD in people aged 30 or above is $3 \%$, and in medical college inpatients it is $6 \%$ (2). The treatment of COPD is mainly focused on relieving the symptoms due to impairment of lung function. There is lack of awareness about the decreased bone mass in COPD patient. COPD itself is a disabling disease, when fracture occurs as a complication of osteoporosis, the quality of life of these patients is further reduced. Therefore there is a great need to screen those patients, so that early diagnosis can be made and preventive and or therapeutic measures can be taken to avoid or reduce the consequences of osteoporosis. A number of techniques have been proposed for the measurement of bone mineral density (BMD) and the diagnosis of osteoporosis. Among them dual energy X-ray absorptiometry (DXA) is an enhanced form of X-ray technology that is used to measure bone loss. It can detect as little as $2 \%$ of bone loss per year. It is a simple, quick and noninvasive procedure. The amount of radiation used is extremely small $(0.01 \mathrm{mSv})$, less than one-tenth the dose $(0.1 \mathrm{mSv})$ of a standard chest X-ray and less than a day's exposure to natural radiation (3). The accuracy of bone mineral density test is high, ranging from $85 \%$ to $99 \%$ (4). Dual energy x-ray 
x-ray absorptiometry (DXA) is currently the most widely available and standardized test, providing rapid, convenient and accurate assessment of BMD. The objective of the study was to evaluate the bone mineral density of the COPD patients and nonCOPD persons and to compare them.

\section{MATERIALS AND METHODS}

In the period of January 2010 to December 2010 this prospective study was carried out in Institute of Nuclear Medicine and Allied Sciences, Barisal. Total 100 subjects were included in the study. Of them, 50 subjects were patients of chronic obstructive pulmonary disease, age ranged from 30 -80 years, diagnosed by respiratory medicine specialist, who were not confined in bed. 50 of them were taken as control group who came in the center for ultrasonography examination for other minor illness that does not affect bone metabolism. The subjects were excluded from the study that had co-existing renal, hepatic, thyroid or celiac disease, tuberculosis, rheumatological, endocrine or metabolic bone disease and any malignancy. The subjects who had been receiving or recently treated with calcium, vitamin D, biphosphonates, levo-thyroxine, lithium, heparin, phenothiazine, methotrexate or androgens were excluded. Pregnant patient were also excluded.

Permission of the study was taken from the concerned departments and authorities. After taking informed written consent, subjects were interviewed and relevant information regarding their disease duration, occupation, life style, nutritional status, smoking history, medications and laboratory values were recorded on a questionnaire form. BMI were also calculated from the height and weight of the patients. All $(n=100)$ subjects underwent DEXA scan of spine and left hip to measure bone mineral density by Lunar DPX Pro (GE) Densitometer. The region of interest was L1- L4 vertebra in spine and femoral neck. Analysis of data from DEXA was computerized and completely automated (SoftwareenCORE 2008, version 12.0). Low bone mass was defined as osteopenia and osteoporosis according to WHO criteria. WHO study group recommended the definition to osteoporosis and osteopenia, based on $\mathrm{BMD}$ value of spine, femoral neck and forearm that expressed as $\mathrm{T}$ score. According to $\mathrm{WHO}$ criteria normal BMD is $\mathrm{T}$ score $\geq-1.0$, osteopenia is $\mathrm{T}$ score $<-1.0$ and $>-2.5$ and osteoporosis is $\mathrm{T}$ score $\leq-2.5$.

Data were processed and analyzed with the help of computer software SPSS (Statistical package for Social Sciences), version 12. Categorical data were expressed as frequencies and corresponding percentages and continuous data were presented as mean, standard deviation (SD). Data presented on categorical form were analyzed using chi-squared test or Fisher's Exact Probability test. While the data presented on continuous scale were analyzed using student's t-test and ANOVA. In each analysis, level of significance was $5 \%$ and $P$ value $<0.05$ was considered significant.

\section{RESULTS}

Fifty COPD patients were in-group $A$ and fifty persons without COPD in control group B. Characteristics of the patients have shown in Table1. Mean BMI of the COPD patient's group (group A) was $19.63 \pm 2.4 \mathrm{~kg} / \mathrm{m}^{2}$ in comparison with 23.34 $\pm 3.8 \mathrm{~kg} / \mathrm{m}^{2}$ in control group (group B). COPD patients (group A) with low BMI had low BMD. Prevalence of low BMD among the study groups has shown in Table 2. COPD patients with prolonged disease had lower BMD than others with shorter disease duration (Table 3).

Among group A, percentage of low BMD was higher $(86.11 \%)$ among steroid users than steroid non-users (64.28\%) (Table 4) but not statistically significant $(p>0.05)$. That suggests bone loss occurs in patients with COPD irrespective of steroid use.

Table 1: Characteristics of the COPD patients (Group A) and Control (Group B)

\begin{tabular}{|l|l|l|}
\hline & Group A $(\mathrm{n}=50)$ & Group B (n=50) \\
\hline Male & $36(72 \%)$ & $39(78 \%)$ \\
\hline Female & $14(28 \%)$ & $11(22 \%)$ \\
\hline Mean Age & $58.2 \pm 11.04$ & $55.3 \pm 11.23$ \\
\hline
\end{tabular}


Table 2: Osteoporosis and Osteopenia between Group A (COPD) and Group B (control):

\begin{tabular}{llll}
\hline $\begin{array}{l}\text { BMD } \\
\text { T score }\end{array}$ & $\begin{array}{l}\text { Group-A } \\
(\mathrm{n}=50)\end{array}$ & $\begin{array}{l}\text { Group-B } \\
(\mathrm{n}=50)\end{array}$ & $\mathrm{p}$ \\
\hline $\begin{array}{l}\text { Lumbar Spine } \\
\text { Normal }\end{array}$ & $7(14)$ & $20(40)$ & \\
Osteopenia & $14(28)$ & $20(40)$ & 0.0002 \\
Osteoporosis & $29(58)$ & $10(20)$ & \\
Femoral Neck & & & \\
Normal & $8(16)$ & $32(64)$ & \\
Osteopenia & $16(32)$ & $12(24)$ & 0.0001 \\
Osteoporosis & $26(52)$ & $6(12)$ & \\
\hline
\end{tabular}

Data presented as No. (\%)

$\mathrm{p}$ value obtained from chi square test.

Table 3: Association of Low BMD and disease duration among Group A:

\begin{tabular}{lllll}
\hline BMD & $\leq 5$ Yrs & $6-10$ Yrs & $\geq 10$ Yrs & $p$ \\
\hline Lumbar Spine & & & & \\
Absolute $\left(\mathrm{g} / \mathrm{cm}^{2}\right)$ & $0.971 \pm 0.11$ & $0.875 \pm 0.15$ & $0.773 \pm 0.15$ & .003 \\
T Score (SD) & $-1.94 \pm 1.02$ & $-2.79 \pm 1.22$ & $-3.63 \pm 1.24$ & .002 \\
Femoral Neck & & & & \\
Absolute (g/cm $\left.{ }^{2}\right)$ & $0.816 \pm 0.15$ & $0.79 \pm 0.16$ & $0.705 \pm 0.15$ & .141 \\
T Score (SD) & $-1.76 \pm 1.25$ & $-2.08 \pm 1.2$ & $-2.68 \pm 1.13$ & .128 \\
\hline
\end{tabular}

Data presented as mean $\pm \mathrm{SD}$.

$p$ value obtained from ANOVA test.

Table 4: Association between steroid use and low BMD in Group A:

\begin{tabular}{llll}
\hline & $\begin{array}{l}\text { Steroid User } \\
(\mathrm{n}=36)\end{array}$ & $\begin{array}{l}\text { Steroid non-user } \\
(\mathrm{n}=14)\end{array}$ & $\mathrm{p}$ \\
Lumbar spine & $31(86.11)$ & $09(64.28)$ & \\
Low BMD & $05(13.88)$ & $05(35.71)$ & 0.11 \\
Normal BMD & & & \\
Femoral neck & $30(83.33)$ & $08(57.14)$ & \\
Low BMD & $06(16.66)$ & $06(42.85)$ & 0.07 \\
Normal BMD & & & \\
\hline
\end{tabular}

Data presented as No (\%)

$\mathrm{p}$ value obtained from Fisher's exact test

\section{DISCUSSION}

Patients with obstructive airway disease have several risk factors that may predispose them to accelerate bone loss and osteoporosis. As airway obstruction worsens, the risk of osteoporosis increases because patients become more debilitated, less mobile, and nutritionally depleted (5). Although osteoporosis is asymptomatic initially, reduced bone mass increases the risk of vertebral, rib, and hip fractures, which can add to the morbidity of obstructive airway disease (6).

In this study, we measured BMD of COPD patients by DXA scan and compared with non-COPD group. Height and weight of each subject were measured and BMI was calculated. BMI was significantly low in group A than group B. This finding correlates with other study (7).

BMD was expressed in absolute value and $\mathrm{T}$ score. Low BMD was defined according to WHO criteria. BMD was significantly low in COPD patients than patients/persons without COPD.

Prevalence of low BMD in COPD patients was $86 \%$ (Osteopenia 28\% and Osteoporosis 58\%) in lumbar spine and $84 \%$ (Osteopenia 32\% and Osteoporosis 52\%) in femoral neck (Table 2 ). This prevalence compared with other studies. Ferguson et al (8) found low BMD in 65\% patients, Jorgensen et al (9) found osteopenia and osteoporosis in 68\% patients and Iqbal et al (7) found $72 \%$ men with low BMD. In other study by Vrieze et al (10) it was 75\%. Prevalence of low BMD in this study was higher than others. This may be due to smaller size, below average nutritional status and less physical exercise of our patients. Different studies also suggest that Asians have slightly lower BMD than whites mainly because of their smaller size (4). Hussain et al. (11) showed significant difference between mean bone mineral density value of young adult population of our country and manufacturer's reference value. Mean BMD value of our population is lower than the supplied data. We do not have a standard reference value of our population, so manufacturer's reference value was used here.

The strength of the present study was, here T score 
was compared with controls from our population. T score is sex matched and ethnic origin dependent, so it is best to compare the BMD values with reference from the same population.

The BMD value was lower than normal in all subgroups of COPD patients but lowest in the group of patients who had prolonged disease ( $\geq 10$ years). This may be due to prolonged duration of the disease or greater mean age of that group. As it showed below normal BMD value in all sub-groups it suggests that COPD itself had an effect for low BMD.

Jorgensen et al. (9) evaluated moderate and severe COPD patients with female predominance. Iqbal et al. (7) studied on moderate and severe COPD and all patients were male. Here patients of both sexes were included, but disease severity could not be classified due to non-availability of Spirometry findings of all patients. Vrieze et al. (10) measured BMD with Quantitative Ultrasonography; here DEXA scan was used for BMD measurement, which is gold standard for osteoporosis diagnosis. The validity and reliability of using bone mineral density measurements from dual-energy absorptiometry are well established.

Here steroid users (either oral or inhaler or both) were compared with only bronchodilator users. Low BMD (osteopenia and osteoporosis) was found in both group of patients but that was not statistically significant (Table 4). BMD was low irrespective of steroid used or not. One limitation of the study is that there are no sufficient details on previous corticosteroids medications, due to very poor record keeping and memory recall by the patients. Systemic steroids adversely affect BMD. The rate of bone loss correlates with daily dose and duration of therapy. However, BMD is reduced in patients with obstructive airway disease who have not treated with corticosteroids, suggesting that other mechanisms are involved in this process (5). Although the effects of inhaled steroids on osteoporosis remain controversial, recent evidence suggests a mild deleterious effect on bone mineral density (12).

Despites some limitations of the study, the findings suggest that the COPD patients have low BMD and that may be due to diseased itself.

\section{CONCLUSION}

Low BMD or osteoporosis and subsequent fracture risk is an important problem in patients with COPD. The glucocorticoid treatment for lung disease is associated with increased risk of bone loss, but the problem is also seen in patients who have not treated with glucocorticoids. The occurrence of fracture as a consequence of low bone mass can cause additional disability to the patients and add the economic burden of the disease. This study showed that bone mineral density is low in chronic obstructive pulmonary disease and dual energy x-ray absorptiometry is an easy and effective method to detect low bone mass. The COPD patients, specially with associated risk factors, such as corticosteroid use, low BMI etc. can be assessed by DXA scan and early diagnosis of low bone mineral density or osteoporosis can be made. Along with the treatment of COPD, the treatment for osteopenia or osteoporosis can be given before they develop fracture. That will increase the quality of life of the COPD patients.

\section{REFERENCES}

1. Innes JA, Reid PT. Respiratory Disease. In: Boon NA, Colledge NR, Walker BR, Hunter JAA eds. (2006). Davidson Principles \& Practice of Medicine. Edinburgh. Churchill Livingstone. 20. p. 647-738.

2. World Health Organization. (2005). Impact of Tobacco related Illness in Bangladesh. World Health Organization. Regional Office Dhaka. January, 2005.

3. Radiological Society of North America. (2010). Patient Safety- X ray: Radiation Exposure in X-ray and $\mathrm{CT}$ Examinations. Available at http://www.radiologyinfo.org

4. Cummings S. R., Bates D, Black, D. M. (2002). Clinical use of bone densitometry. JAMA. 288. p. 1889-1897.

5. Biskobing D M. (2002). COPD and osteoporosis. Chest. 121. p. 609-620.

6. NIH Consensus Development Panel on Osteoporosis Prevention, Diagnosis, and Therapy. 2001. Osteoporosis prevention, diagnosis and therapy. JAMA. 285. p. 785-795. 
7. Iqbal F, Michaelson J, Thaler L, Rubin J, Roman J, Nanes MS. (1999). Declining bone mass in men with chronic pulmonary disease: contribution of glucocorticoid treatment, body mass index, and gonadal function. Chest. 116. p. 1616-1624.

8. Ferguson GT, Calverley PM, Anderson JA, Jenkins CR, Jones PW, Willits LA, et al. (2009). Prevalence and Progression of Osteoporosis is Patients with COPD. Chest. 136. p. $1456-1465$.

9. Jorgensen NR, Schwarz P, Holme I, Henriksen BM, Petersen LJ, Backer V. (2007). The prevalence of osteoporosis in patients with chronic obstructive pulmonary disease: a cross sectional study. Respir Med. 2007. 101. p. 177-185.

10. Vrieze A, de Greef MH, Wijkstra PJ, Wempe JB. (2007). Low bone mineral density in COPD patients related to worse lung function, low weight and decreased fat-free mass. Osteoporos Int. 18. p. 1197 1202.

11. Hussain FA, Nissa L, Haq M. (2010). Standardization of bone mineral density (BMD) for Bangladeshi population- A preliminary study. Bangladesh Journal of Nuclear Medicine. 13(1). p. 16-19.

12. Wong CA, Walsh LJ. (2000). Smith CJP. Inhaled corticosteroid use and bone-mineral density in patients with asthma. The Lancet. 355. p.1399-1403. 